Archive for

Organic Chemistry

Arkivoc 2020, part viii, 46-57

\title{
Synthesis and characterization of some new S-substituted sulfanylpyridines, thieno[2,3-b]pyridines and related heterocycles
}

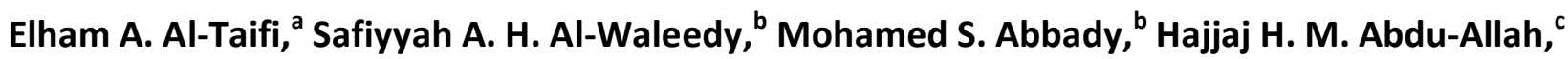 \\ Islam S. Marae, ${ }^{\text {b }}$ Suzan Abuelhassan, ${ }^{\text {b }}$ and Etify A. Bakhite ${ }^{\text {b* }}$ \\ ${ }^{a}$ Department of Chemistry, Faculty of Science, Sana'a University, Sana'a, Yemen \\ ${ }^{b}$ Department of Chemistry, Faculty of Science, Assiut University, Assiut, Egypt \\ ${ }^{c}$ Department of Pharmaceutical Organic Chemistry, Faculty of Pharmacy, Assiut University, Egypt \\ Email: betiafy@yahoo.com
}

Accepted 10-11-2020

Published on line 10-26-2020

\section{Abstract}

Ethyl (3-cyano-5-ethoxycarbonyl-6-methyl-4-styryl-2-pyridylsulfanyl)acetate was prepared and reacted with hydrazine hydrate in ethanol to give a mixture of (3-cyano-5-ethoxycarbonyl-6-methyl-4-styryl-2pyridylsulfanyl)acetohydrazide and diethyl 3-amino-6-methyl-4-styrylthieno[2,3-b]pyridine-2,5-dicarboxylate. The latter compound was reacted with 2,5-dimethoxytetrahydrofuran to give 3-(1H-pyrrol-1-yl)thieno[2,3b]pyridine analogue which on treatment with hydrazine hydrate in ethanol furnished 5-ethoxycarbonyl-6methyl-3-(1H-pyrrol-1-yl)-4-styrylthieno[2,3-b]pyridine-2-carbohydrazide. Both acetohydrazide and carbohydrazide were used as precursors for the title compounds.<smiles>CCOC(=O)c1c(C)nc2sc3c(=O)n([Al])cnc3c2c1/C=C/c1ccccc1</smiles>

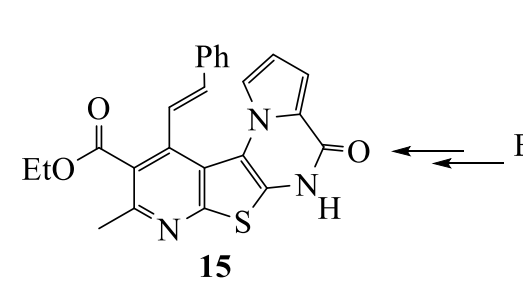<smiles>CCOC(=O)c1c(C)nc2sc(C(=O)NN)c(-n3cccc3)c2c1/C=C/c1ccccc1</smiles><smiles>CCOC(=O)c1c(C)nc(SCC(=O)NN)c(C#N)c1/C=C/c1ccccc1</smiles><smiles>CCOC(=O)CSc1nc(C)c(C(=O)OCC)c(/C=C/c2ccccc2)c1C#N</smiles>

Keywords: Fused pyridines, thiophenes, pyrimidines, pyrroles 


\section{Introduction}

Pyridine and its derivatives abundantly exist in nature and became interesting targets in 1930 with the importance of niacin for the treatment of dermatitis and dementia. ${ }^{1}$ Some pyridines have been reported to exhibit good biological activities and medicinal applications, and numbers of them are in clinical uses. ${ }^{2}$ Many thieno[2,3-b]pyridines have been synthesized and reported to show versatile biological and pharmacological applications. $^{3,4}$ Some of them proved to possess antiviral, ${ }^{5,6}$ anti-diabetic, ${ }^{7}$ antimicrobial, ${ }^{8-11}$ antiinflammatory, ${ }^{12,13}$ antitumor, ${ }^{14,15}$ anticancer, ${ }^{16}$ antiparasitic ${ }^{17}$ and neurotropic activities. ${ }^{18}$ Others are useful as sedatives, ${ }^{19}$ gonadotropin releasing hormone antagonists, ${ }^{20,21}$ anticoagulants, ${ }^{22}$ anti-atherosclerotics ${ }^{23}$ and as analgesics. ${ }^{24,25}$ In view of the aforementioned facts and as a continuation of our programme towards synthesis of new heterocyclic compounds with expected biological and medicinal applications, ${ }^{26}$ this work was planned to synthesize and explore the synthetic utility of both (3-cyano-5-ethoxycarbonyl-6-methyl-4-styryl-2pyridylsulfanyl)acethydrazide (4) and 5-ethoxycarbonyl-6-methyl-3-(1H-pyrrol-1-yl)-4-styrylthieno[2,3-b]pyridine-2-carbohydrazide (10) hoping to get new compounds with potential pharmacological and biological applications.

\section{Results and Discussion}

The starting compound, ethyl 3-cyano-1,2-dihydro-6-methyl-4-styryl-2-thioxopyridine-5-carboxylate (1) was prepared according to our previous method (Scheme 1). ${ }^{26}$ Reaction of 1 with ethyl chloroacetate by refluxing in ethanol containing slightly excess amounts of sodium acetate for 30 minutes produced the expected ester, ethyl (3-cyano-5-ethoxycarbonyl-6-methyl-4-styryl-2-pyridylsulfanyl)acetate (2). When the above reaction was repeated increasing the reaction time to 3 hours, the product was identified as diethyl 3-amino-6-methyl-4styrylthieno[2,3-b]pyridine-2,5-dicarboxylate (3). However, cyclization of $\mathbf{2}$ to $\mathbf{3}$ was achieved by heating in ethanol containing a catalytic quantity of sodium acetate for 2.5 hours (Scheme 1).

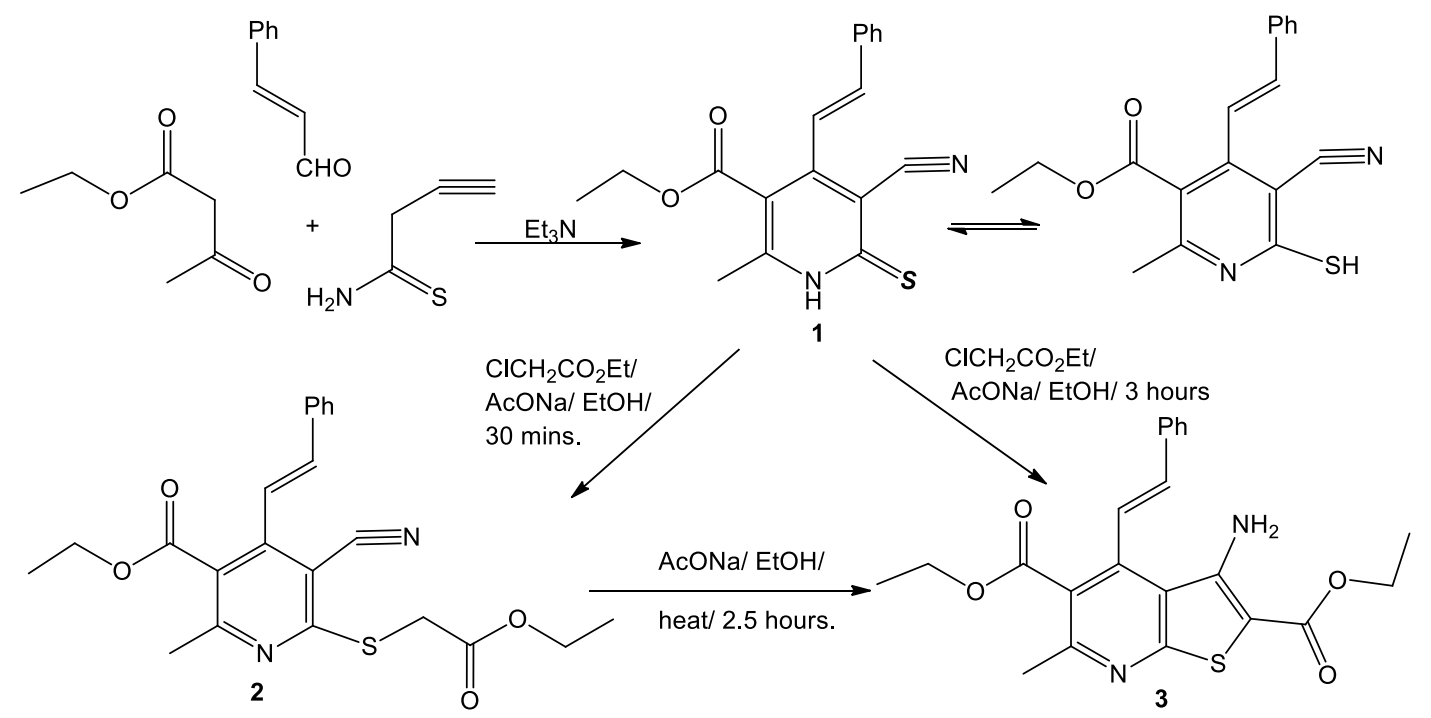

Scheme 1. Synthesis of compounds 1-3. 
The physical properties of compounds $\mathbf{1}^{26}$ and $\mathbf{3}^{27}$ are in agreement with those previously reported. IR spectrum of 2 showed characteristic absorption bands at 2219 for $(C \equiv N)$, at 1748 for $(C=O$, non-conjugated ester) and at 1724 for $\left(\mathrm{C}=\mathrm{O}\right.$, conjugated ester). ${ }^{1} \mathrm{H}$ NMR spectrum 2 (DMSO- $d_{6}$ ) displayed a multiplet at $\delta 6.60$ $7.63\left(7 \mathrm{H}: \mathrm{CH}=\mathrm{CH}\right.$ and $\mathrm{Ar}-\mathrm{H}$ 's), a multiplet at $\delta 4.16-4.37\left(6 \mathrm{H}\right.$ : two $\mathrm{OCH}_{2}$ and $\left.\mathrm{SCH}_{2}\right)$, a singlet at $\delta 2.52\left(3 \mathrm{H}, \mathrm{CH}_{3}\right.$ at C-6, overlapped with solvent signal) and a multiplet at $\delta 1.21-1.27$ (6H: two $\mathrm{CH}_{3}$ of ester groups).

Heating an ethanolic solution of the ester $\mathbf{2}$ with hydrazine hydrate furnished a mixture of (3-cyano-5ethoxycarbonyl-6-methyl-4-styryl-2-pyridylsulfanyl)acetohydrazide (4) and diethyl 3-amino-6-methyl-4-styrylthieno[2,3-b]pyridine-2,5-dicarboxylate (3) (Scheme 2).

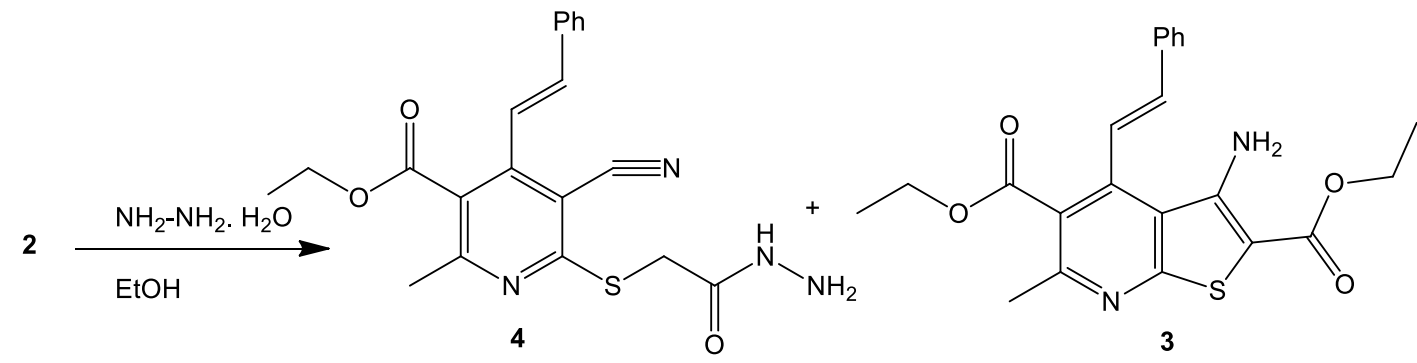

Scheme 2. Synthesis of compounds 3 and 4.

IR spectrum of 4 showed characteristic absorption bands at 3321, 3210, 3105 for $\left(\mathrm{NHNH}_{2}\right), 3027$ for $(\mathrm{C}-\mathrm{H}$, aromatic), 2986 for ( $\mathrm{C}-\mathrm{H}$, aliphatic), 2218 for $(\mathrm{C} \equiv \mathrm{N}), 1717$ for $(\mathrm{C}=\mathrm{O})$ and 1651 for ( $\mathrm{C}=\mathrm{O}$, hydrazide).

$\mathrm{H}$ NMR spectrum of $4\left(\mathrm{CDCl}_{3}\right)$ displayed a singlet at $\delta 7.92(1 \mathrm{H}, \mathrm{NH})$, a multiplet at $\delta 7.09-7.52(7 \mathrm{H}: \mathrm{CH}=\mathrm{CH}$ and Ar-H's), a quartet at $\delta 4.36-4.41\left(2 \mathrm{H}, \mathrm{OCH}_{2}\right)$, a singlet at $\delta 3.91\left(2 \mathrm{H}, \mathrm{SCH}_{2}\right)$, a broad singlet at $\delta 3.89(2 \mathrm{H}$, $\left.\mathrm{NH}_{2}\right)$, a singlet at $\delta 2.61\left(3 \mathrm{H}, \mathrm{CH}_{3}\right.$ at C-6) and a triplet at $\delta 1.30-1.34\left(3 \mathrm{H}, \mathrm{CH}_{3}\right.$ of ester $)$.

${ }^{13} \mathrm{C} N M R$ and Dept 135 spectra of $4\left(\mathrm{CDCl}_{3}\right)$ showed the following peaks: $\delta 169.90,167.21,161.90,158.89$, 148.01, $140.54(\mathrm{CH}), 135.14,129.72(\mathrm{CH}), 128.93(\mathrm{CH}), 127.42(\mathrm{CH}), 126.70,124.50,120.35(\mathrm{CH}), 114.42$, 102.95, $62.14\left(\mathrm{OCH}_{2}\right), 31.97\left(\mathrm{SCH}_{2}\right), 23.37\left(\mathrm{CH}_{3}\right.$ at $\left.\mathrm{C}-6\right), 14.14 \mathrm{CH}_{3}$ of ester group) which are in agreement with its structure.

Reaction of acetohydrazide 4 with phenyl isothiocyanate gave the corresponding thiosemicarbazide derivative 5. Condensation of acetohydrazide 4 with some aromatic aldehydes or thiophene-2-carboxaldehyde afforded $N^{\prime}$-arylmethylene or (thien-2-yl)methylene)(3-cyano-5-ethoxycarbonyl-6-methyl-4-styryl-2-pyridylsulfanyl)acetohydrazides $\mathbf{6 a - d}$. Cyclization of $\mathbf{6 a - d}$ into the corresponding 3-amino- $N^{\prime}$-arylmethylene or (thien-2-yl)methylene-5-ethoxycarbonyl-6-methyl-4-styrylthieno[2,3-b]pyridine-2-carbohydrazides 7a-d was achieved by heating in ethanol containing catalytic amounts of sodium ethoxide. Treatment of 7a-d with triethyl orthoformate in the presence of acetic anhydride produced 3-arylmethylene or (thien-2-yl)methylene aminopyridothienopyrimidine-4(3H)-ones 8a-d in high yield (Scheme 3).

It is noteworthy that the intramolecular cyclizations of compounds $\mathbf{2}$ and $\mathbf{6 a - \mathbf { d }}$ to the corresponding thieno[2,3-b]pyridines $\mathbf{3}$ and $\mathbf{7 a - d}$ obey Thorpe-Ziegler reaction which its mechanism is reviewed before. ${ }^{4}$ 


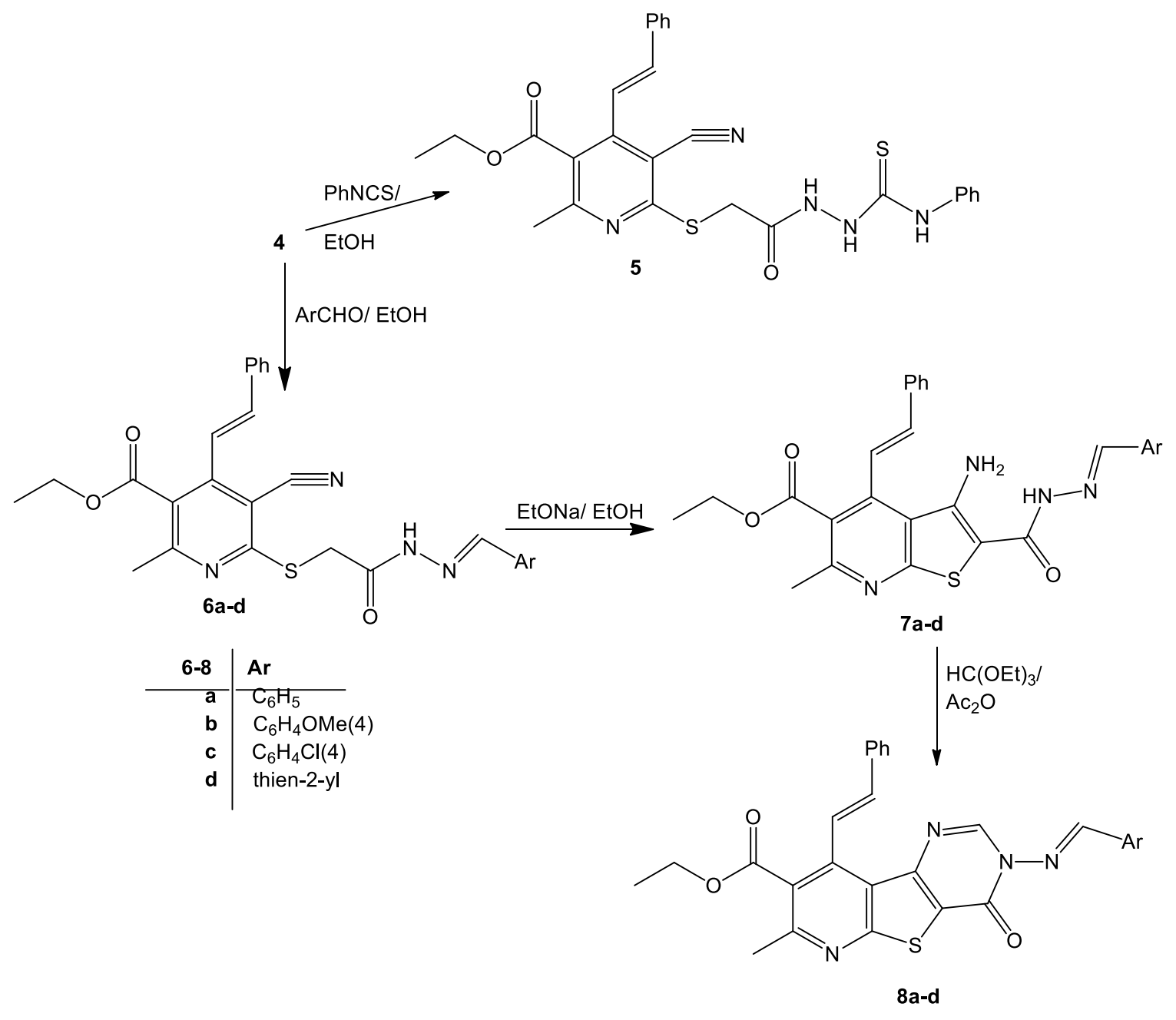

Scheme 3. Synthesis of compounds 5, 6a-d, 7a-d and 8a-d.

IR spectrum of 5 exhibited three absorption bands in the region 3280 to 3200 characteristic for (NH) groups. Its ${ }^{1}$ HNMR spectrum showed three singlet signals at $\delta$ values $9.80,9.19$ and 8.40 corresponding to three $(\mathrm{NH})$ groups beside the other signals. IR spectra of 6a-d showed characteristic absorption bands in the regions 3190 to 3208 for $(\mathrm{NH}), 2221$ to 2224 for $(\mathrm{C}=\mathrm{N}), 1719$ to 1725 for (C=O, ester) and 1655 to 1679 for ( $\mathrm{C}=\mathrm{O}$, acetohydrazide). Their ${ }^{1} \mathrm{H}$ NMR spectra showed a singlet at $\delta$ value ranged from 10.12 to 11.48 corresponds to $(\mathrm{NH})$ group and a singlet signal at $\delta$ value ranged from 4.15 to 4.57 equivalent to $\mathrm{SCH}_{2} \mathrm{group}_{\text {. }}$ IR spectra of compounds 7a-d showed characteristic absorption bands in the regions 3450 to 3461,3303 to 3311, 3215 to 3132 for $\left(\mathrm{NH}_{2}\right)$ and $(\mathrm{NH})$ groups, 1716 to 1724 for $\left(\mathrm{C}=\mathrm{O}\right.$, ester) and 1628 to $1640{ }^{1}$ for $(\mathrm{C}=\mathrm{O}$, carbohydrazide). Their ${ }^{1} \mathrm{HNMR}$ spectra exhibited a singlet signal at $\delta$ value ranged from 9.51 to 10.85 corresponds to $(\mathrm{NH})$ group and a singlet signal at $\delta$ value ranged from 6.74 to 6.93 equivalent to $\mathrm{NH}_{2}$ group. IR spectra of compounds 8a-d showed characteristic absorption bands in the regions 1705 to 1670 for (C=O, ester) and 1670 to 1687 for ( $C=0$, pyrimidinone). Their ${ }^{1} \mathrm{H}$ NMR spectra exhibited a singlet at $\delta$ value ranged from 9.26 to 9.63 corresponds to $(\mathrm{CH})$ of pyrimidinone moiety. ${ }^{13} \mathrm{C}$ NMR and Dept 135 spectral data of $6 a$ and $\mathbf{6 c}$ and $\mathbf{7 b}$ are in accordance with their structures.

Incorporating the pyrrole nucleus into thieno[2,3-b]pyridine framework was achieved by reacting compound 3 with 2,5-dimethoxytetrahydrofuran, when the pyrrolyl derivative 9 was obtained. Heating compound $\mathbf{9}$ with hydrazine hydrate in ethanol for two hours resulted in partial hydrazinolysis and formation 
of 5-ethoxycarbonyl-6-methyl-3-(1H-pyrrol-1-yl)-4-styrylthieno[2,3-b]pyridine-2-carbohydrazide (10) in high yield. Condensation of carbohydrazide $\mathbf{1 0}$ with some aromatic aldehydes by refluxing in ethanol yielded the corresponding acylhydrazones 11a-c. Diazotization of carbohydrazide 10 led to 5-ethoxycarbonyl-6-methyl-3(1H-pyrrol-1-yl)-4-styrylthieno[2,3-b]pyridine-2-carbonyl azide (12) (Scheme 4).<smiles>COC1CCC(OC)O1</smiles><smiles>CCOC(=O)c1c(C)nc2sc(C(=O)OCC(=O)OCC(N)=O)c(C(=O)OCC)c2c1-n1cccc1</smiles>

9
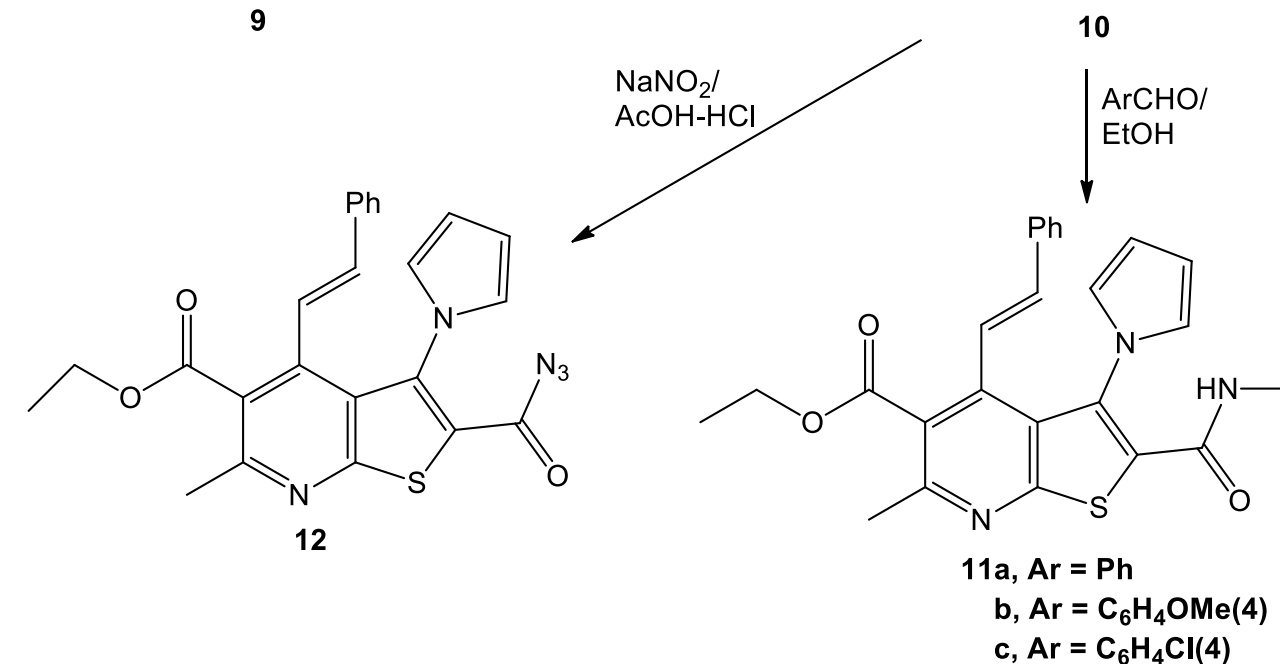

Scheme 4. Synthesis of compounds 9, 10, 11a-c and 12.

The IR spectrum of 9 revealed the disappearance of $\mathrm{V}_{\mathrm{NH} 2}$. Its ${ }^{1} \mathrm{H}$ NMR spectrum showed a doublet at $\delta$ value ranged from 6.94 to 6.95 for $2 \mathrm{CH}$ (pyrrole) and a doublet signal at $\delta$ value 6.22 for $2 \mathrm{CH}$ (pyrrole). IR spectrum of compound 10 showed absorption bands at 3418, 3334, 3278 for $\left(\mathrm{NHNH}_{2}\right), 1723$ for $(\mathrm{C}=\mathrm{O}$, ester group attached to pyridine ring) and 1657 for $\left(\mathrm{C}=\mathrm{O}\right.$, carbohydrazide). Its ${ }^{1} \mathrm{HNMR}$ spectrum showed a singlet at $\delta 8.06(\mathrm{NH})$ and a singlet signal at $\delta 4.53\left(\mathrm{~s}, 2 \mathrm{H}, \mathrm{NH}_{2}\right)$. IR spectra of compounds 11a-c showed an absorption band in the regions 3290 to 3299 for $(\mathrm{NH}), 1716-1724$ for $(\mathrm{C}=\mathrm{O}$, ester) and 1664-1673 for (C=O, carbohydrazide). IR spectrum of compound 12 showed absorption bands at 2142 for $\left(N_{3}\right), 1724$ for $(C=0$, ester) and 1691 for ( $C=0$, carbonylazide). MS of compounds $\mathbf{9 ,} 10$ and $\mathbf{1 1 b}$ are in agreement with their structures.

Heating carbonylazide $\mathbf{1 2}$ in ethanol at reflux temperature for two hours provided a mixture of the corresponding carbamate 14 and the fused pyrazinone 15. This reaction may proceed via Curtius rearrangement of the carbonyl azide $\mathbf{1 3}$ affording the reactive isocyanate intermediate 13 which underwent in situ either reaction with ethanol giving the carbamate $\mathbf{1 4}$, or an intramolecular cycloaddition reaction 
affording pyrazinone 15, respectively (Scheme 5). In contrast, heating carbonylazide 12 in an inert solvent such as toluene furnished pyrazinone $\mathbf{1 5}$ as the sole product (Scheme 5).

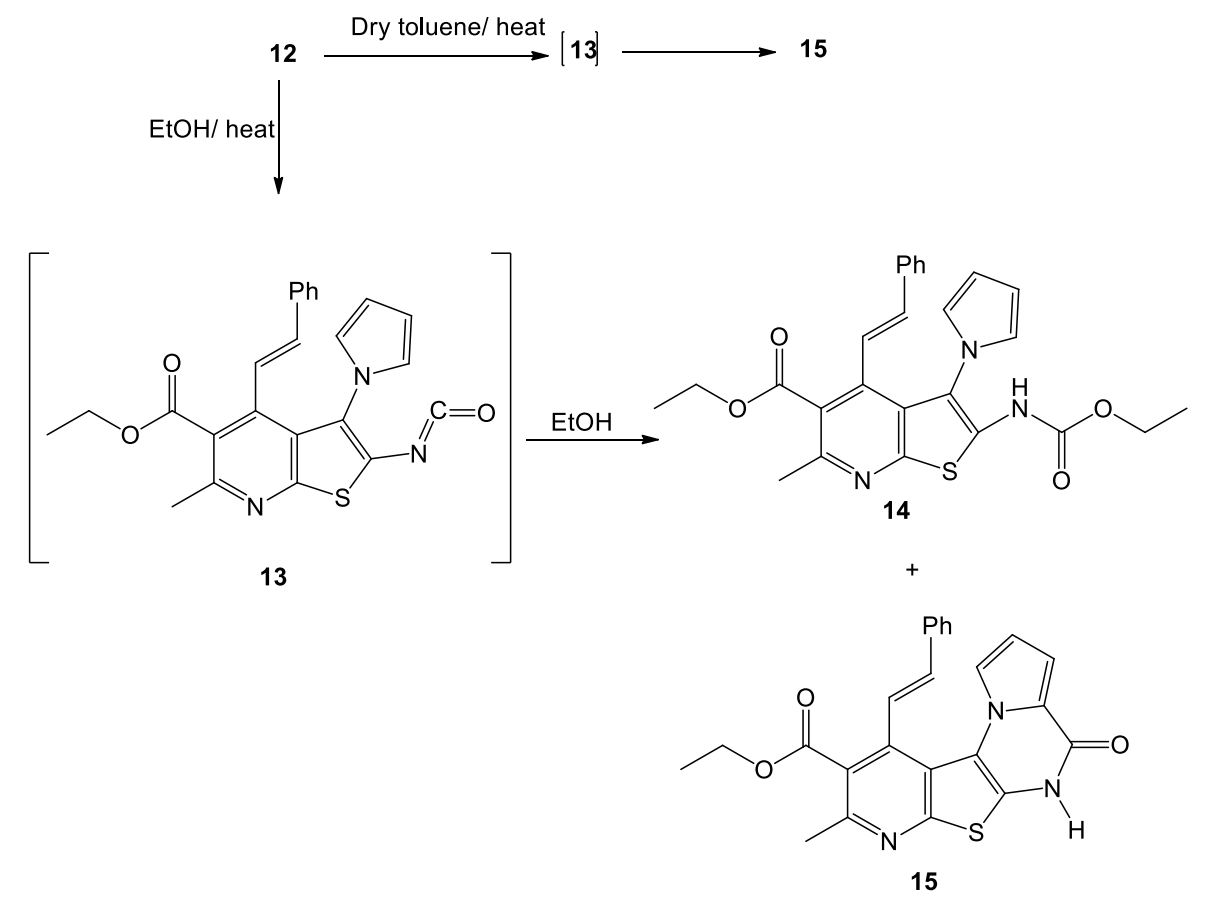

Scheme 5. Synthesis of compounds 14 and 15.

IR spectrum of 14 showed absorption bands at $3411(\mathrm{NH}), 1728$ for $(\mathrm{C}=\mathrm{O}$, ester) and 1669 for $(\mathrm{C}=\mathrm{O}$, carbamate). IR spectrum of 15 showed absorption bands at 3243 for (NH), 1719 for (C=O, ester) and 1644 for ( $C=0$, pyrazinone). ${ }^{1} \mathrm{H}$ NMR spectra of compounds $\mathbf{1 4}$ and $\mathbf{1 5}$ are in accordance with their structures.

It is noteworthy that the double bond of the styryl group (2-phenylethenyl group) of all prepared compounds possesses E-configuration since we start with E-cinnamaldehyde and all entire sequence of reactions took place far away from this double bond. This fact was further supported via our previous publication $^{27}$ in which the crystal structure of compound 3 was studied and proved the E-configuration of the styryl group.

\section{Conclusions}

(3-Cyano-5-ethoxycarbonyl-6-methyl-4-styryl-2-pyridylsulfanyl)acetohydrazide (4) and 5-ethoxycarbonyl-6methyl-3-(1H-pyrrol-1-yl)-4-styrylthieno[2,3-b]pyridine-2-carbohydrazide (10) have been synthesized and successfully used as precursors for synthesizing other new $S$-substituted sulfanylpyridines, thienopyridines, pyrrolylthienopyridines and pyridothienopyrimidines as well as pyridolthienopyrrolopyrazinones. Full characterization of all these compounds is reported. The newly synthesized compounds are interesting for their potential pharmacological and biological applications owing to their incorporation of additional pharmacophores. 


\section{Experimental Section}

General. Melting points were determined on a Gallenkamp apparatus. The IR spectra were recorded on a Shimadzu $470 \mathrm{IR}$-spectrophotometer $\left(\mathrm{KBr} ; v_{\max }\right.$ in $\left.\mathrm{cm}^{-1}\right)$. The ${ }^{1} \mathrm{H}$ NMR spectra were recorded on a Bruker 400 $\mathrm{MHz}$ spectrometer using $\mathrm{CDCl}_{3}$ or DMSO- $d_{6}$ as a solvent and tetramethylsilane (TMS) as internal reference. ${ }^{13} \mathrm{C}$ NMR and Dept 135 spectra were recorded on on a Bruker $100 \mathrm{MHz}$ spectrometer using $\mathrm{CDCl}_{3}$ or DMSO- $d_{6}$ as a solvent and tetramethylsilane (TMS) as internal reference. Coupling constants (J values) are given in Hertz $(\mathrm{Hz})$. Elemental analyses were performed on Perkin Elmer 2400 LS Series CHN/O analyzer. MS analyses were performed on a Thermo Scientific single quadrupole mass spectrometer Model: ISQ 7000.

Ethyl 3-cyano-1,2-dihydro-6-methyl-4-styryl-2-thioxopyridine-5-carboxylate (1). Compound 1 was synthesized according to our previous method. ${ }^{26}$

Ethyl (3-cyano-5-ethoxycarbonyl-6-methyl-4-styryl-2-pyridinyl-sulfanyl)acetate (2). A mixture of 1 (6.48 g, 20 $\mathrm{mmol})$, ethyl chloroacetate $(2.2 \mathrm{~mL}, 20 \mathrm{mmol})$ and sodium acetate trihydrate $(3.0 \mathrm{~g}, 22 \mathrm{mmol})$ in ethanol (70 $\mathrm{mL}$ ) was refluxed for 30 mins. The solid that formed after dilution with water $(30 \mathrm{~mL})$ was filtered off and crystallized from methanol as fine colorless crystals of 2. Yield: $7.00 \mathrm{~g} \mathrm{(85 \% );} \mathrm{mp} \mathrm{70-71}{ }^{\circ} \mathrm{C}$. Anal. Calcd. For $\mathrm{C}_{22} \mathrm{H}_{22} \mathrm{~N}_{2} \mathrm{O}_{4} \mathrm{~S}$ (410.13): C, 64.37; $\mathrm{H}, 5.40 ; \mathrm{N}, 6.82 \%$. Found: $\mathrm{C}, 64.12 ; \mathrm{H}, 5.34 ; \mathrm{N}, 6.91 \%$.

Diethyl 3-amino-6-methyl-4-styrylthieno[2,3-b]pyridine-2,5-dicarboxylate (3). Method A: A mixture of 2 (2.05 $\mathrm{g}, 5 \mathrm{mmol})$ and sodium acetate trihydrate $(0.14 \mathrm{~g}, 1 \mathrm{mmol})$ in ethanol $(25 \mathrm{~mL})$ was heated under reflux for for 2.5 hours. The product that formed after cooling was filtered off, washed with water and crystallized from ethanol to give compound 3; yield: $1.90 \mathrm{~g}$ (93\%); mp $116-117^{\circ} \mathrm{C}$ ( Lit. $\left.^{25} 116-117{ }^{\circ} \mathrm{C}\right)$

Method B: A mixture of $1(3.24 \mathrm{~g}, 10 \mathrm{mmol})$, ethyl chloroacetate $(1.1 \mathrm{~mL}, 10 \mathrm{mmol})$ and sodium acetate trihydrate $(1.50 \mathrm{~g}, 11 \mathrm{mmol})$ in ethanol $(25 \mathrm{~mL})$ was refluxed for 3 hours. The product that obtained upon recrystallization was identical to that described above in all aspects.

Reaction of ethyl (3-cyano-5-ethoxycarbonyl-6-methyl-4-styryl-2-pyridylsulfanyl)acetate (2) with hydrazine hydrate. A mixture of ester $2(4.10 \mathrm{~g}, 10 \mathrm{mmol})$ and hydrazine hydrate $(0.5 \mathrm{~mL}, 10 \mathrm{mmol})$ in ethanol $(40 \mathrm{~mL})$ was refluxed for 10 mins. The white sold which formed while hot was filtered off and crystallized from dioxane as white needles. This product was assigned as (3-cyano-5-ethoxycarbonyl-6-methyl-4-styryl-2pyridylsulfanyl)acethydrazide (4). Yield: $1.10 \mathrm{~g}(28 \%)$; mp 222-223 ${ }^{\circ} \mathrm{C}$. Anal. Calcd. For $\mathrm{C}_{20} \mathrm{H}_{20} \mathrm{~N}_{4} \mathrm{O}_{3} \mathrm{~S}$ (396.13): C, $60.59 ; \mathrm{H}, 5.08 ; \mathrm{N}, 14.13 \%$. Found: $\mathrm{C}, 60.84 ; \mathrm{H}, 5.11 ; \mathrm{N}, 14.00 \%$.

The filtrate of the above crude product was allowed to cool whereby fine yellow needles formed. These were filtered off and recrystallized from ethanol. This compound was identified as diethyl 3-amino-6-methyl-4styrylthieno[2,3-b]pyridine-2,5-dicarboxylate (3). Yield: $2.05 \mathrm{~g}$ (50\%).

$\boldsymbol{N}^{1}$-(3-Amino-5-ethoxycarbonyl-6-methyl-4-styrylthieno[2,3-b]pyridine-2-carbonyl)- $\boldsymbol{N}^{4}$-phenylthiosemicarbazide (5). A mixture of $4(1.98 \mathrm{~g}, 5 \mathrm{mmol})$ and phenyl iso-thiocyanate $(0.70 \mathrm{~mL})$ in ethanol $(30 \mathrm{~mL}) \mathrm{was}$ refluxed for one hour. The white product was filtered off and crystallized from dioxane to give needle crystals of 5. Yield: $2.13 \mathrm{~g}(80 \%) ; \mathrm{mp} 287-288^{\circ} \mathrm{C}$. IR $\left(\mathrm{cm}^{-1}\right): 3280,3143(3 \mathrm{NH}), 3002(\mathrm{CH}$, aromatic), $2224(\mathrm{C} \equiv \mathrm{N}), 1719$ (C=O, ester), $1678\left(\mathrm{C}=\mathrm{O}\right.$, amide). ${ }^{1} \mathrm{H}$ NMR (DMSO-d $)$ : $\delta 9.80(\mathrm{~s}, 1 \mathrm{H}, \mathrm{NH}), 9.19(\mathrm{~s}, 1 \mathrm{H}, \mathrm{NH}), 8.40(\mathrm{~s}, 1 \mathrm{H}, \mathrm{NH}), 7.10-$ $8.10\left(\mathrm{~m}, 12 \mathrm{H}: \mathrm{CH}=\mathrm{CH}\right.$ and $\left.\mathrm{Ar}-\mathrm{H}^{\prime} \mathrm{s}\right), 4.36\left(\mathrm{q}, 2 \mathrm{H}, \mathrm{OCH}_{2}\right), 4.13\left(\mathrm{~s}, 2 \mathrm{H}, \mathrm{SCH}_{2}\right), 2.58\left(\mathrm{~s}, 3 \mathrm{H}, \mathrm{CH}_{3}\right.$ at C-6), 1.30-1.33 (t, $3 \mathrm{H}, \mathrm{CH}_{3}$ of ester ). Anal. Calcd. For $\mathrm{C}_{27} \mathrm{H}_{25} \mathrm{~N}_{5} \mathrm{O}_{3} \mathrm{~S}_{2}$ (531.14): C, 61.00; $\mathrm{H}, 4.74 ; \mathrm{N}, 13.17$ \%. Found: $\mathrm{C}, 61.35 ; \mathrm{H}$, $4.78 ; \mathrm{N}, 13.04 \%$.

Condensation of acetohydrazide 4 with aromatic aldehydes or thiophene-2- carboxaldehyde; formation of hydrazone derivatives 6a-d; general procedure. A mixture of acetohydrazide 4 (3.96 g, $10 \mathrm{mmol}$ ) and the 
appropirate aldehyde $(11 \mathrm{mmol})$ in ethanol $(40 \mathrm{~mL})$ was refluxed for $3 \mathrm{~h}$. The precipitated product was filtered off and recrystallized from dioxane to give white crystals of 6a-d.

N'-Benzylidene(3-cyano-5-ethoxycarbonyl-6-methyl-4-styryl-2-pyridylsulfanyl)acethydrazide (6a). Compound 6a was obtained from compound 4 and benzaldehyde. Yield: $4.11 \mathrm{~g}(85 \%) ; \mathrm{mp} 161-162{ }^{\circ} \mathrm{C}$. IR $\left(\mathrm{cm}^{-1}\right): 3192$ (NH), 3002 (CH, aromatic), 2877 ( $\mathrm{CH}$, aliphatic), $2222(\mathrm{C} \equiv \mathrm{N}), 1724\left(\mathrm{C}=\mathrm{O}\right.$, ester), 1659 (C=O, hydrazide). ${ }^{1} \mathrm{H} N M R$ $\left(\mathrm{CDCl}_{3}\right): \delta 10.43(\mathrm{~s}, 1 \mathrm{H}, \mathrm{NH}), 8.12(\mathrm{~s}, 1 \mathrm{H}, \mathrm{N}=\mathrm{CH}), 7.02-7.86\left(\mathrm{~m}, 12 \mathrm{H}: \mathrm{CH}=\mathrm{CH}\right.$ and $\left.\mathrm{Ar}-\mathrm{H}^{\prime} \mathrm{s}\right), 4.29(\mathrm{~s}, 2 \mathrm{H}, \mathrm{SCH}), 4.31-$ 4.35 (q, $\left.2 \mathrm{H}, \mathrm{OCH}_{2}\right), 2.48\left(\mathrm{~s}, 3 \mathrm{H}, \mathrm{CH}_{3}\right.$ at C-6), 1.28-1.30 (t, 3H, $\mathrm{CH}_{3}$ of ester ). ${ }^{13} \mathrm{C} \mathrm{NMR}$ and Dept $135\left(\mathrm{CDCl}_{3}\right)$ : $170.69,166.85,164.54,161.99,158.60,148.83(\mathrm{CH}), 147.53,144.95(\mathrm{CH}), 140.67(\mathrm{CH}), 135.35,133.54,130.37$ $(\mathrm{CH}), 129.67(\mathrm{CH}), 128.80(\mathrm{CH}), 127.41(\mathrm{CH}), 123.75,120.31(\mathrm{CH}), 114.45,102.38,62.07\left(\mathrm{OCH}_{2}\right), 32.21\left(\mathrm{SCH}_{2}\right)$, $23.49\left(\mathrm{CH}_{3}\right.$ at C-6), $14.14\left(\mathrm{CH}_{3}\right.$ of ester group). Anal. Calcd. For $\mathrm{C}_{27} \mathrm{H}_{24} \mathrm{~N}_{4} \mathrm{O}_{3} \mathrm{~S}$ (484.15): $\mathrm{C}, 66.92 ; \mathrm{H}, 4.99 ; \mathrm{N}$, $11.56 \%$. Found: $\mathrm{C}, 66.78 ; \mathrm{H}, 4.73 ; \mathrm{N}, 11.39 \%$.

\section{N'-(4-Methoxybenzylidene)(3-cyano-5-ethoxycarbonyl-6-methyl-4-styryl-2-pyridylsulfanyl)acethydrazide}

(6b). Compound 6b was obtained from compound 4 and 4-methoxybenzaldehyde. Yield: $4.62 \mathrm{~g}(90 \%) ; \mathrm{mp}$ 195-196 ${ }^{\circ} \mathrm{C}$. IR $\left(\mathrm{cm}^{-1}\right): 3190(\mathrm{NH}), 3001\left(\mathrm{CH}\right.$, aromatic), $2224(\mathrm{C} \equiv \mathrm{N}), 1722\left(\mathrm{C}=\mathrm{O}\right.$, ester), $1655\left(\mathrm{C}=\mathrm{O}\right.$, hydrazide). ${ }^{1} \mathrm{H}$ NMR (DMSO- $\left.d_{6}\right): \delta 11.48(\mathrm{~s}, 1 \mathrm{H}, \mathrm{NH}), 8.00(\mathrm{~s}, 1 \mathrm{H}, \mathrm{N}=\mathrm{CH}), 7.00-7.63(\mathrm{~m}, 11 \mathrm{H}: \mathrm{CH}=\mathrm{CH}$ and Ar-H's), 4.15-4.59 (m, $4 \mathrm{H}: \mathrm{OCH}_{2}$ and $\left.\mathrm{SCH}_{2}\right), 3.82\left(\mathrm{~s}, 3 \mathrm{H}, \mathrm{OCH}_{3}\right), 2.51\left(\mathrm{~s}, 3 \mathrm{H}, \mathrm{CH}_{3}\right.$ at C-6), 1.23-1.26 (t, 3H, $\mathrm{CH}_{3}$ of ester). Anal. Calcd. for $\mathrm{C}_{28} \mathrm{H}_{26} \mathrm{~N}_{4} \mathrm{O}_{4} \mathrm{~S}$ (514.17): C, 65.35; H, 5.09; N, 10.89\%. Found: C,65.15; H, 5.22; N, 10.64\%.

N'-(4-Chlorobenzylidene)(3-cyano-5-ethoxycarbonyl-6-methyl-4-styryl-2-pyridylsulfanyl)acethydrazide (6c). Compound 6c was obtained from compound 4 and 4-chlorobenzaldehyde. Yield: $4.71 \mathrm{~g} \mathrm{(91 \% );} \mathrm{mp} \mathrm{200-202}{ }^{\circ} \mathrm{C}$. IR $\left(\mathrm{cm}^{-1}\right)$ : $3208(\mathrm{NH}), 3000(\mathrm{CH}$, aromatic), 2985 ( $\mathrm{CH}$, aliphatic), $2221(\mathrm{C} \equiv \mathrm{N}), 1719$ (C=O, ester), 1659 (C=O, hydrazide). ${ }^{1} \mathrm{H}$ NMR $\left(\mathrm{CDCl}_{3}\right): \delta 11.36(\mathrm{~s}, 1 \mathrm{H}, \mathrm{NH}), 8.21(\mathrm{~s}, 1 \mathrm{H}, \mathrm{N}=\mathrm{CH}), 7.11-7.96\left(\mathrm{~m}, 11 \mathrm{H}: \mathrm{CH}=\mathrm{CH}\right.$ and $\left.\mathrm{Ar}-\mathrm{H}^{\prime} \mathrm{s}\right)$, $4.57\left(\mathrm{~s}, 2 \mathrm{H}, \mathrm{SCH}_{2}\right), 4.32-4.36$ (q, $\left.2 \mathrm{H}, \mathrm{OCH}_{2}\right), 2.52$ (s, 3H, $\mathrm{CH}_{3}$ at C-6), 1.30-1.33 (t, 3H, $\mathrm{CH}_{3}$ of ester). ${ }^{13} \mathrm{C}$ NMR and Dept $135\left(\mathrm{CDCl}_{3}\right): \delta 169.66,167.01,162.71,158.47,147.33,146.79,142.69,139.58,135.88,135.17,132.69$, $129.59,128.83,128.14,127.22,123.56,120.67,114.81,102.17,61.97\left(\mathrm{OCH}_{2}\right), 32.86\left(\mathrm{SCH}_{2}\right), 23.40\left(\mathrm{CH}_{3}\right.$ at $\left.\mathrm{C}-6\right)$, $14.02\left(\mathrm{CH}_{3}\right.$ of ester). Anal. Calcd. for $\mathrm{C}_{27} \mathrm{H}_{23} \mathrm{ClN}_{4} \mathrm{O}_{3} \mathrm{~S}$ (518.12): C, 62.48; $\mathrm{H}, 4.47 ; \mathrm{N}, 10.79 \%$. Found:_C, 62.71; $\mathrm{H}$, $4.44 ; \mathrm{N}, 10.84 \%$.

N'-(2-Thienylmethylene)-(3-cyano-5-ethoxycarbonyl-6-methyl-4-styryl-2-pyridylsulfanyl)acethydrazide (6d). Compound $6 \mathrm{~d}$ was obtained from 4 and thiophene-2-carboxaldehyde. Yield: $4.30 \mathrm{~g}(88 \%)$; mp_210-211 ${ }^{\circ} \mathrm{C}$. IR $\left(\mathrm{cm}^{-1}\right)$ : $3198(\mathrm{NH}), 3084,3027(\mathrm{CH}$, aromatic), 2981, $2883(\mathrm{CH}$, aliphatic), 2223 (C=N), 1725 (C=O, ester), 1664 $\left(\mathrm{C}=\mathrm{O}\right.$, acetohydrazide). ${ }^{1} \mathrm{H}$ NMR $\left(\mathrm{CDCl}_{3}\right): \delta 10.12(\mathrm{~s}, 1 \mathrm{H}, \mathrm{NH}), 7.12-7.62(\mathrm{~m}, 11 \mathrm{H}: \mathrm{CH}=\mathrm{CH}, \mathrm{N}=\mathrm{CH}$, thiophene-H and Ar-H's), 4.34-4.36 (q, 2H, OCH $\left.{ }_{2}\right), 4.23\left(\mathrm{~s}, 2 \mathrm{H}, \mathrm{SCH}_{2}\right), \delta 2.51\left(\mathrm{~s}, 3 \mathrm{H}, \mathrm{CH}_{3}\right), 1.24-1.26\left(\mathrm{t}, 3 \mathrm{H}, \mathrm{CH}_{3}\right.$ of ester). Anal. Calcd. for $\mathrm{C}_{25} \mathrm{H}_{22} \mathrm{~N}_{4} \mathrm{O}_{3} \mathrm{~S}_{2}$ (490.11): C, 61.21; $\mathrm{H}, 4.52 ; \mathrm{N}, 11.42 \%$. Found $\mathrm{C}, 61.00 ; \mathrm{H}, 4.39 ; \mathrm{N}, 11.61 \%$.

Cyclization of ylideneacethydrazides 6a-d; formation of thieno[2,3-b]pyridines 7a-d; general procedure. To a suspension of $6 \mathrm{a}-\mathbf{d}(5 \mathrm{mmol})$ in ethanol $(25 \mathrm{~mL})$, an ethanolic sodium ethoxide solution $(0.15 \mathrm{~g}$ of sodium in 40 $\mathrm{mL}$ of absolute ethanol) was added. The resulting mixture was refluxed for 10 mins. The precipitated solid was filtered off and recrystallized from dimethylsulfoxide to give 7a-d.

3-Amino- $N$ '-benzylidene-5-ethoxycarbonyl-6-methyl-4-styrylthieno[2,3-b]pyridine-2-carbohydrazide (7a). Compound 7a was synthesized from 6a. Yield: $2.32 \mathrm{~g}(92 \%) ; \mathrm{mp} 280-282{ }^{\circ} \mathrm{C}$. IR $\left(\mathrm{cm}^{-1}\right): 3461,3303,3150\left(\mathrm{NH}_{2}\right.$, $\mathrm{NH}), 2929$ (C-H, aliphatic), 1716 (C=O, ester), 1634 (C=O, carbohydrazide). ${ }^{1} \mathrm{H}$ NMR (DMSO- $\left.d_{6}\right): \delta 10.85$ (s, $1 \mathrm{H}$, $\mathrm{NH}), 7.22-7.98\left(\mathrm{~m}, 13 \mathrm{H}: \mathrm{N}=\mathrm{CH}, \mathrm{CH}=\mathrm{CH}\right.$ and $\mathrm{Ar}-\mathrm{H}$ 's), $6.91\left(\mathrm{~s}, 2 \mathrm{H}, \mathrm{NH}_{2}\right), 4.32-4.36\left(\mathrm{q}, 2 \mathrm{H}, \mathrm{OCH}_{2}\right), 2.56\left(\mathrm{~s}, 3 \mathrm{H}, \mathrm{CH}_{3}\right.$ at C-6), 1.26-1.29 (t, 3H, CH $\mathrm{CH}_{3}$ of ester ). Anal. Calcd. For $\mathrm{C}_{27} \mathrm{H}_{24} \mathrm{~N}_{4} \mathrm{O}_{3} \mathrm{~S}$ (484.16): C, 66.92; $\mathrm{H}, 4.99 ; \mathrm{N}, 11.56 \%$. Found: $\mathrm{C}, 67.08 ; \mathrm{H}, 4.74 ; \mathrm{N}, 11.33 \%$. 
3-Amino-N' -(4-methoxybenzylidene)-5-ethoxycarbonyl-6-methyl-4-styrylthieno[2,3-b]pyridine-2-carbohydrazide (7b). Compound $7 \mathrm{~b}$ was synthesized from $6 \mathrm{~b}$. Yield: $2.43 \mathrm{~g}(95 \%) ; \mathrm{mp} 248-250{ }^{\circ} \mathrm{C} . \mathrm{IR}\left(\mathrm{cm}^{-1}\right): 3457$, 3300, $3153\left(\mathrm{NH}_{2}, \mathrm{NH}\right), 2929$ ( $\mathrm{CH}$, aliphatic), 1716 (C=O, ester), 1632 (C=0, hydrazide). ${ }^{1} \mathrm{H} N M R\left(D M S O-d_{6}\right): \delta$ $9.91(\mathrm{~s}, 1 \mathrm{H}, \mathrm{NH}), 6.90-7.80\left(\mathrm{~m}, 12 \mathrm{H}: \mathrm{N}=\mathrm{CH}, \mathrm{CH}=\mathrm{CH}\right.$ and $\left.\mathrm{Ar}-\mathrm{H}^{\prime} \mathrm{s}\right), 6.84\left(\mathrm{~s}, 2 \mathrm{H}, \mathrm{NH}_{2}\right), 4.30-4.34\left(\mathrm{q}, 2 \mathrm{H}, \mathrm{OCH}_{2}\right), 3.83$ $\left(\mathrm{s}, 3 \mathrm{H}, \mathrm{OCH}_{3}\right), 2.64\left(\mathrm{~s}, 3 \mathrm{H}, \mathrm{CH}_{3}\right.$ at $\left.\mathrm{C}-6\right), 1.25-1.28\left(\mathrm{t}, 3 \mathrm{H}, \mathrm{CH}_{3}\right.$ of ester ). ${ }^{13} \mathrm{C} \mathrm{NMR}$ and Dept 135 (DMSO-d $\left.d_{6}\right): \delta$ $170.28,168.41,167.20,162.74,161.14,156.00,144.23,141.52,139.67,137.88,135.33,129.60,128.88$, 127.38, 126.53, 125.51, 121.56, 120.78, 114.16, 95.86, $62.01\left(\mathrm{OCH}_{2}\right), 55.34\left(\mathrm{OCH}_{3}\right), 23.44\left(\mathrm{CH}_{3}\right.$ at C-6), 14.24 $\left(\mathrm{CH}_{3}\right.$ of ester). Anal. Calcd. For $\mathrm{C}_{28} \mathrm{H}_{26} \mathrm{~N}_{4} \mathrm{O}_{4} \mathrm{~S}$ (514.17): C,65.35; H, 5.09; N, 10.89\%. Found: C,65.15; H, 5.00; N, $10.72 \%$.

3-Amino- $N^{\prime}$-(4-chlorobenzylidene)-5-ethoxycarbonyl-6-methyl-4-styrylthieno[2,3-b]pyridine-2-carbo-

hydrazide (7c). Compound 7c was synthesized from 6c. Yield: $2.48 \mathrm{~g}(95 \%) ; \mathrm{mp} 285-287^{\circ} \mathrm{C} . \mathrm{IR}\left(\mathrm{cm}^{-1}\right): 3450$, 3311, $3215\left(\mathrm{NH}_{2}, \mathrm{NH}\right), 2929\left(\mathrm{CH}\right.$, aliphatic), 1720 (C=O, ester), $1640\left(\mathrm{C}=\mathrm{O}\right.$, carbohydrazide). ${ }^{1} \mathrm{H} \mathrm{NMR}\left(\mathrm{CDCl}_{3}\right): \delta$ $10.56(\mathrm{~s}, 1 \mathrm{H}, \mathrm{NH}), 7.10-8.06\left(\mathrm{~m}, 12 \mathrm{H}: \mathrm{N}=\mathrm{CH}, \mathrm{CH}=\mathrm{CH}\right.$ and $\left.\mathrm{Ar}-\mathrm{H}^{\prime} \mathrm{s}\right), 6.93\left(\mathrm{~s}, 2 \mathrm{H}, \mathrm{NH}_{2}\right), 4.34-4.38(\mathrm{q}, 2 \mathrm{H}, \mathrm{OCH}), 2.57$ (s, 3H, $\mathrm{CH}_{3}$ at C-6), 1.28-1.31 (t, 3H, $\mathrm{CH}_{3}$ of ester ). Anal. Calcd. for $\mathrm{C}_{27} \mathrm{H}_{23} \mathrm{ClN}_{4} \mathrm{O}_{3} \mathrm{~S}$ (519.02): C, 62.48; $\mathrm{H}, 4.47 ; \mathrm{N}$, $10.79 \%$. Found: $\mathrm{C}, 62.16 ; \mathrm{H}, 4.49 ; \mathrm{N}, 10.62 \%$.

3-Amino- $N^{\prime}$-(2-thienylmethylene)-5-ethoxycarbonyl-6-methyl-4-styrylthieno[2,3-b]pyridine-2-carbo-

hydrazide (7d). Compound 7d was synthesized from $6 \mathrm{~d}$. Yield: $2.15 \mathrm{~g}(87 \%) ; \mathrm{mp} 280-281^{\circ} \mathrm{C} . \mathrm{IR}\left(\mathrm{cm}^{-1}\right): 3452$, 3304, $3132\left(\mathrm{NH}_{2}, \mathrm{NH}\right)$, 3060, 3023 (CH, aromatic), 2957, 2923 ( $\mathrm{CH}$, aliphatic), 1724 (C=O, ester), 1628 (C=0, carbohydrazide). ${ }^{1} \mathrm{H}$ NMR $\left(\mathrm{CDCl}_{3}\right):{ }^{1} \mathrm{H}$ NMR $\left(\mathrm{DMSO}_{-} \mathrm{d}_{6}\right): \delta 9.51(\mathrm{~s}, 1 \mathrm{H}, \mathrm{NH}), 7.74-7.78(\mathrm{~d}, 1 \mathrm{H}, \mathrm{CH}=\mathrm{C}), 7.33-7.68(\mathrm{~m}$, $9 \mathrm{H}: \mathrm{N}=\mathrm{CH}$, thiophene-H and $\left.\mathrm{Ar}-\mathrm{H}^{\prime} \mathrm{s}\right), 6.80-6.84(\mathrm{~d}, 1 \mathrm{H}, \mathrm{C}=\mathrm{CH}), 6.74\left(\mathrm{~s}, 2 \mathrm{H}, \mathrm{NH}_{2}\right), 4.26-4.29\left(\mathrm{q}, 2 \mathrm{H}, \mathrm{OCH}_{2}\right), 2.60(\mathrm{~s}$, $3 \mathrm{H}, \mathrm{CH}_{3}$ at C-6), 1.18-1.20 (t, 3H, $\mathrm{CH}_{3}$ of ester). Anal. Calcd. for $\mathrm{C}_{25} \mathrm{H}_{22} \mathrm{~N}_{4} \mathrm{O}_{3} \mathrm{~S}_{2}$ (490.11): $\mathrm{C}, 61.21 ; \mathrm{H}, 4.52 ; \mathrm{N}$, $11.42 \%$. Found $C, 61.33 ; \mathrm{H}, 4.46 ; \mathrm{N}, 11.50 \%$.

\section{3-Arylmethyleneamino-8-ethoxycarbonyl-7-methyl-9-styrylpyrido[3',2':4,5]thieno[3,2-d]pyrimidin-4(3H)-}

ones (8a-d); general procedure. A mixture of $7 \mathrm{a}-\mathrm{d}(2 \mathrm{mmol})$ and $\mathrm{HC}(\mathrm{OEt})_{3}(5 \mathrm{~mL})$ in acetic anhydride $(10 \mathrm{~mL})$ was refluxed for $4 \mathrm{~h}$. The precipitated solid was filtered off and crystallized from isopropanol as white crystals of 8a-d.

\section{3-Benzylideneamino-8-ethoxycarbonyl-7methyl-9-styrylpyrido[3',2':4,5]thieno[3,2-d]pyrimidine-4(3H)-one}

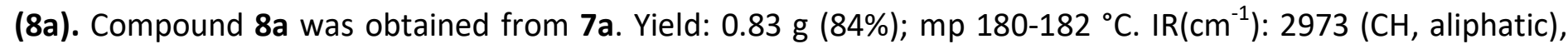
1705 ( $\mathrm{C}=\mathrm{O}$, ester), 1670 ( $\mathrm{C}=\mathrm{O}$, pyrimidinone). ${ }^{1} \mathrm{H}$ NMR (DMSO-d 6 ): $\delta 10.00(\mathrm{~s}, 1 \mathrm{H}$, pyrimidinone- $\mathrm{H}$ ), $9.60(\mathrm{~s}, 1 \mathrm{H}$, $\mathrm{N}=\mathrm{CH}$ ), 7.24-8.04 (m, 12H: $\mathrm{N}=\mathrm{CH}, \mathrm{CH}=\mathrm{CH}$ and $\mathrm{Ar}-\mathrm{H}$ 's), 4.31-4.35 (q, 2H, $\left.\mathrm{OCH}_{2}\right), 2.57\left(\mathrm{~s}, 3 \mathrm{H}, \mathrm{CH}_{3}\right.$ at C-6), 1.23-1.2 (t, 3H, $\mathrm{CH}_{3}$ of ester). Anal. Calcd. For $\mathrm{C}_{28} \mathrm{H}_{22} \mathrm{~N}_{4} \mathrm{O}_{3} \mathrm{~S}$ (494.14): C, 68.00; $\mathrm{H}, 4.48 ; \mathrm{N}, 11.33 \%$. Found: $\mathrm{C}, 67.82 ; \mathrm{H}$, 4.47; N, $11.19 \%$.

3-(4-Methoxybenzylideneamino)-8-ethoxycarbonyl-7methyl-9-styrylpyrido[3',2':4,5]thieno[3,2-d]pyrimidin4(3H)-one (8b). Compound 8b was obtained from 7b. Yield: $0.92 \mathrm{~g}(88 \%) ; \mathrm{mp} 188-190{ }^{\circ} \mathrm{C}$. IR $\left(\mathrm{cm}^{-1}\right): 2977(\mathrm{CH}$, aliphatic), 1719 ( $\mathrm{C}=\mathrm{O}$, ester), 1678 ( $\mathrm{C}=\mathrm{O}$, pyrimidinone). ${ }^{1} \mathrm{H}$ NMR (DMSO- $\left.d_{6}\right): \delta 9.46$ (s, $1 \mathrm{H}, \mathrm{pyrimidinone-H)}$ ), $9.22(\mathrm{~s}, 1 \mathrm{H}, \mathrm{N}=\mathrm{CH}), 6.87-7.92(\mathrm{~m}, 11 \mathrm{H}: \mathrm{CH}=\mathrm{CH}$ and $\mathrm{Ar}-\mathrm{H} ' \mathrm{~s}), 4.31-4.34\left(\mathrm{q}, 2 \mathrm{H}, \mathrm{OCH}_{2}\right), 3.81\left(\mathrm{~s}, 3 \mathrm{H}, \mathrm{OCH}_{3}\right), 2.66(\mathrm{~s}$, $3 \mathrm{H}, \mathrm{CH}_{3}$ at C-6), 1.24-1.27 (t, 3H, $\mathrm{CH}_{3}$ of ester ). Anal. Calcd. For $\mathrm{C}_{29} \mathrm{H}_{24} \mathrm{~N}_{4} \mathrm{O}_{4} \mathrm{~S}$ (524.15): $\mathrm{C}, 66.40 ; \mathrm{H}, 4.61 ; \mathrm{N}$, $10.68 \%$. Found: $\mathrm{C}, 66.59 ; \mathrm{H}, 4.73 ; \mathrm{N}, 10.52 \%$.

3-(4-Chlorobenzylideneamino)-8-ethoxycarbonyl-7-methyl-9-styrylpyrido[3',2':4,5]thieno[3,2-d]pyrimidin4(3H)-one (8c). Compound 8c was obtained from 7c. Yield: $0.93 \mathrm{~g}(88 \%) ; \mathrm{mp} \mathrm{220-222}{ }^{\circ} \mathrm{C} . \mathrm{IR}\left(\mathrm{cm}^{-1}\right): 3021(\mathrm{CH}$, aromatic), $2981\left(\mathrm{CH}\right.$, aliphatic), 1721 ( $\mathrm{C}=\mathrm{O}$, ester), 1687 (C=O, pyrimidinone). ${ }^{1} \mathrm{H}$ NMR (DMSO- $\left.d_{6}\right): \delta 9.75(\mathrm{~s}, 1 \mathrm{H}$, $\mathrm{CH}$ pyrimidinone), $9.50(\mathrm{~s}, 1 \mathrm{H}, \mathrm{N}=\mathrm{CH}), 6.55-8.07\left(\mathrm{~m}, 11 \mathrm{H}: \mathrm{CH}=\mathrm{CH}\right.$ and $\left.\mathrm{Ar}-\mathrm{H}^{\prime} \mathrm{s}\right), 4.00 .4 .04\left(\mathrm{q}, 2 \mathrm{H}, \mathrm{OCH}_{2}\right), 2.74(\mathrm{~s}$, 
$3 \mathrm{H}, \mathrm{CH}_{3}$ at C-6), 1.16-1.19 (t, 3H, $\mathrm{CH}_{3}$ of ester). Anal. Calcd. for $\mathrm{C}_{28} \mathrm{H}_{21} \mathrm{ClN}_{4} \mathrm{O}_{3} \mathrm{~S}$ (528.10): C, 63.57; $\mathrm{H}, 4.00 ; \mathrm{N}$, $10.59 \%$. Found: C, 63.42; H, 4.12; N, $10.43 \%$.

3-(2-Thienylmethyleneamino)-8-ethoxycarbonyl-7-methyl-9-styrylpyrido[3',2':4,5]thieno[3,2-d]pyrimidin4(3H)-one (8d). Compound 8d was obtained from 7d. Yield: $0.95 \mathrm{~g}(95 \%) ; \mathrm{mp} \mathrm{202-204}{ }^{\circ} \mathrm{C}$. IR $\left(\mathrm{cm}^{-1}\right): 3063,3025$ ( $\mathrm{CH}$, aromatic), 2983, $2933\left(\mathrm{CH}\right.$, aliphatic), 1720 (C=O, ester), $1673\left(\mathrm{C}=0\right.$, pyrimidinone). ${ }^{1} \mathrm{H} \mathrm{NMR}\left(\mathrm{CDCl}_{3}\right): \delta$ 8.45-8.49 (d, J $16 \mathrm{~Hz}, 1 \mathrm{H}, \mathrm{CH}=\mathrm{C}), 8.26(\mathrm{~s}, 1 \mathrm{H}, \mathrm{CH}$ pyrimidinone), 7.28-7.60 (m, 9H: N=CH, thiophene-H and ArH's), 7.07-7.11 (d, J $16 \mathrm{~Hz}, 1 \mathrm{H}, \mathrm{C}=\mathrm{CH}), 4.41$ (q, 2H, OCH$)_{2}, 2.77$ (s, 3H, $\mathrm{CH}_{3}$ at C-6), 1.26-1.35 (t, 3H, $\mathrm{CH}_{3}$ of ester). Anal. Calcd. for $\mathrm{C}_{26} \mathrm{H}_{20} \mathrm{~N}_{4} \mathrm{O}_{3} \mathrm{~S}_{2}$ (500.10): C, 62.38; $\mathrm{H}, 4.03 ; \mathrm{N}, 11.19 \%$. Found: $\mathrm{C}, 62.21 ; \mathrm{H}, 4.06 ; \mathrm{N}, 11.00 \%$.

Diethyl 6-methyl-3-(1H-pyrrol-1-yl)-4-styrylthieno[2,3-b]pyridine-2,5-dicarboxylate (9). A mixture of ester 4 $(3.96 \mathrm{~g}, 10 \mathrm{mmol})$ and 2,5-dimethoxytetrahydrofuran $(1.84 \mathrm{~mL}, 14 \mathrm{mmol})$ in glacial acetic acid $(30 \mathrm{~mL}) \mathrm{was}$ refluxed for $3 \mathrm{~h}$. The precipitate that formed was filtered off and recrystallized from ethanol to give colorless needles of 9. Yield: $4.15 \mathrm{~g}$ (90\%); m.p.: 127-128 ${ }^{\circ} \mathrm{C}$. IR ( $\left.\mathrm{cm}^{-1}\right)$ : 3094, 3061, 3024 (C-H, aromatic), 2991,2900 (C$\mathrm{H}$, aliphatic), $1722\left(\mathrm{C}=\mathrm{O}\right.$, attached to pyridine ring), 1694 ( $\mathrm{C}=\mathrm{O}$, attached to thiophene ring). ${ }^{1} \mathrm{H}$ NMR (DMSO$\left.d_{6}\right)$ : $\delta$ 7.30-7.33 (m, 3H, Ar-H's), 7.16-7.18 (m, 2H, Ar-H's); 6.94-6.95 (d, 2H, pyrrole-H), 6.69-6.73 (d, $\left.1 \mathrm{H}, \mathrm{CH}=\mathrm{C}\right)$; 6.20-6.23 (m, 3H: C=CH and Pyrrole-H); 4.25-4.29 (q, 2H, OCH 2$), 4.13-4.17\left(q, 2 \mathrm{H}, \mathrm{OCH}_{2}\right), 2.50\left(\mathrm{~s}, 3 \mathrm{H}, \mathrm{CH}_{3}\right.$ at C6), 1.15-1.18 (t, 3H, $\mathrm{CH}_{3}$ of ester); 1.19-1.22 (t, 3H, $\mathrm{CH}_{3}$ of ester). MS: at $\mathrm{m} / \mathrm{z} 460\left(\mathrm{M}^{+}, 15 \%\right), 415$ (M $\mathrm{M}^{+-}$OEt, 100\%). Anal. Calcd. for $\mathrm{C}_{26} \mathrm{H}_{24} \mathrm{~N}_{2} \mathrm{O}_{4} \mathrm{~S}$ (460.15): C, 67.81; $\mathrm{H}, 5.25 ; \mathrm{N}, 6.08 \%$. Found: $\mathrm{C}, 67.59 ; \mathrm{H}, 5.27 ; \mathrm{N}, 6.00 \%$

5-Ethoxycarbonyl-6-methyl-3-(1H-pyrrol-1-yl)-4-styrylthieno[2,3-b]pyridine-2-carbohydrazide (10). A mixture of ester $9(2.30 \mathrm{~g}, 5 \mathrm{mmol})$ and hydrazine hydrate $(0.5 \mathrm{~mL}, 10 \mathrm{mmol})$ in ethanol $(40 \mathrm{~mL})$ was refluxed for $3 \mathrm{~h}$. The solid that formed was filtered off and crystallized from dioxane as white needles of 10. Yield: $2.06 \mathrm{~g}$ (92\%); m.p.: $230-231^{\circ} \mathrm{C} . \mathrm{IR}\left(\mathrm{cm}^{-1}\right):$ 3418, 3334, $3278\left(\mathrm{NHNH}_{2}\right), 2996,2899(\mathrm{C}-\mathrm{H}$, aliphatic), 1723 (C=O, ester), 1657 (C=O, carbohydrazide). ${ }^{1} \mathrm{H}$ NMR (DMSO- $\left.d_{6}\right): \delta 8.06(\mathrm{~s}, 1 \mathrm{H}, \mathrm{NH}), 6.19-7.33(11 \mathrm{H}: \mathrm{CH}=\mathrm{CH}, \mathrm{pyrrole}-\mathrm{H}, \mathrm{Ar}-\mathrm{H} ' \mathrm{~s}), 4.53$ $\left.\left(\mathrm{s}, 2 \mathrm{H}, \mathrm{NH}_{2} \text { ), 4.26-4.29 (q, 2H, OCH}\right)_{2}\right), 2.62$ (s, 3H, $\mathrm{CH}_{3}$ at C-6), 1.15-1.18 (t, 3H, $\mathrm{CH}_{3}$ of ester). MS: $\mathrm{m} / \mathrm{z} 446\left(\mathrm{M}^{+}\right.$, $30 \%), 401\left(\mathrm{M}^{+-}-\mathrm{OEt}, 100 \%\right), 415\left(\mathrm{M}^{+-}-\mathrm{NHNH}_{2}, 80 \%\right)$. Anal. Calcd. for $\mathrm{C}_{24} \mathrm{H}_{22} \mathrm{~N}_{4} \mathrm{O}_{3} \mathrm{~S}(446.14): \mathrm{C}, 64.56 ; \mathrm{H}, 4.97$; $\mathrm{N}, 12.55 \%$. Found: C, 64.71; H, 4.87; N,12.32\%.

Condensation of carbohydrazide 10 with aromatic aldehydes; Synthesis of pyrrolylthieno[2,3-b]pyridines 11a-c; general procedure. A mixture of $10(0.89 \mathrm{~g}, 2 \mathrm{mmol})$ and the respective aromatic or heterocyclic aldehyde $(2 \mathrm{mmol})$ in ethanol $(25 \mathrm{~mL})$ was refluxed for $2 \mathrm{~h}$. The precipitate that formed while hot was filtered off and recrystallized from dioxane to give white crystals of 11a-c.

N'-Benzylidene-5-ethoxycarbonyl-6-methyl-3-(1H-pyrrol-1-yl)-4-styrylthieno[2,3-b]pyridine-2-carbohydrazide (11a). Compound 11a was prepared from 10 and benzaldehyde. Yield: $1.00 \mathrm{~g} \mathrm{(93 \% );} \mathrm{mp} \mathrm{258-259}{ }^{\circ} \mathrm{C}$. IR $\left(\mathrm{cm}^{-1}\right)$ : $3292(\mathrm{NH})$, 3058, $3027(\mathrm{CH}$, aromatic), $2981(\mathrm{CH}$, aliphatic), $1716(\mathrm{C}=\mathrm{O}$, ester), $1664(\mathrm{C}=\mathrm{O}$, carbohydrazide). ${ }^{1} \mathrm{H}$ NMR (DMSO- $\left.d_{6}\right): \delta 7.15-7.30(\mathrm{~m}, 11 \mathrm{H}: \mathrm{N}=\mathrm{CH}$ and Ar-H's); 6.90-6.92 (d, 2H, pyrrole-H), 6.70-6.73 (d, $1 \mathrm{H}, \mathrm{CH}=\mathrm{C}) ; 6.20-6.24\left(\mathrm{~m}, 3 \mathrm{H}: \mathrm{C}=\mathrm{CH}\right.$ and pyrrole-H); 4.26-4.30 (q, 2H, $\left.\mathrm{OCH}_{2}\right) ; 2.60\left(\mathrm{~s}, 3 \mathrm{H}, \mathrm{CH}_{3}\right.$ at C6), 1.12-1.15(t, 3H, $\mathrm{CH}_{3}$ of ester). Anal. Calcd. For $\mathrm{C}_{31} \mathrm{H}_{26} \mathrm{~N}_{4} \mathrm{O}_{3} \mathrm{~S}$ (534.17): C, 69.64; $\mathrm{H}, 4.90 ; \mathrm{N}, 10.48 \%$. Found: $\mathrm{C}$, 69.33; $\mathrm{H}, 4.88 ; \mathrm{N}, 10.30 \%$.

$N^{\prime}$-(4-Methoxybenzylidene)-5-ethoxycarbonyl-6-methyl-3-(1H-pyrrol-1-yl)-4-styrylthieno[2,3-b]pyridine-2carbohydrazide (11b). Compound 11b was prepared from 10 and 4-methoxybenzaldehyde. Yield: $1.00 \mathrm{~g}(88$ \%); mp 224-225 ${ }^{\circ} \mathrm{C}$. IR $\left(\mathrm{cm}^{-1}\right): 3299(\mathrm{NH}), 3061(\mathrm{CH}$, aromatic), 2986, 2927, 2836 (CH, aliphatic), 1719 (C=O, ester), 1669 ( $\mathrm{C}=\mathrm{O}$, carbohydrazide). MS: $\mathrm{m} / z 564$ (M+, 20\%); 134 (100\%). Anal. Calcd. For $\mathrm{C}_{32} \mathrm{H}_{28} \mathrm{~N}_{4} \mathrm{O}_{4} \mathrm{~S}(564.18)$ : C,68.07; H, 5.00; N, 9.92\%. Found: C,68.00; H, 5.09; N, 9.82\%.

$N^{\prime}$-(4-Chlorobenzylidene)-5-ethoxycarbonyl-6-methyl-3-(1H-pyrrol-1-yl)-4-styrylthieno[2,3-b]pyridine-2carbohydrazide (11c). Compound 11c was prepared from 10 and 4-chlorobenzaldehyde. Yield: 1.02 g (89\%); 


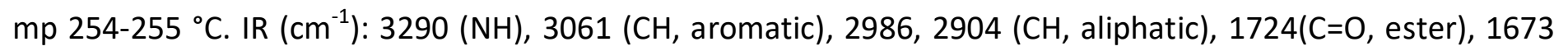
( $C=0$, carbohydrazide). Anal. Calcd. for $\mathrm{C}_{31} \mathrm{H}_{25} \mathrm{ClN}_{4} \mathrm{O}_{3} \mathrm{~S}$ (568.13): C, 65.43; $\mathrm{H}, 4.43 ; \mathrm{N}, 9.85 \%$. Found: $\mathrm{C}, 65.70 ; \mathrm{H}$, $4.44 ; \mathrm{N}, 9.63 \%$.

5-Ethoxycarbonyl-6-methyl-3-(1H-pyrrol-1-yl)-4-styrylthieno[2,3-b]pyridine-2-carbonylazide (12). To a cold solution of compound 4 (3.96 g, $10 \mathrm{mmol})$ in glacial acetic acid $(30 \mathrm{~mL})$, a cold solution of sodium nitrite (0.69 g; $10 \mathrm{mmol}$ in $2 \mathrm{~mL}$ water) was added dropwise with stirring. After the completion of addition, stirring was continued for 1 hour at room temperature. The solid that formed was collected by filtration, washed abundantly with cold water and air dried. It was applied in the next step without purification; Yield: $4.10 \mathrm{~g}$ (89\%); mp 140-141 ${ }^{\circ} \mathrm{C}$ (dec.). IR $\left(\mathrm{cm}^{-1}\right)$ : 2982 (CH, aliphatic), $2142\left(\mathrm{~N}_{3}\right), 1724$ (C=O, ester), 1691(C=0, carbonylazide).

Heating carbonylazide 12 in ethanol; Formation of ethylcarbamate 14 and pyrazinone 15 . Compound 12 $(0.91 \mathrm{~g}, 2 \mathrm{mmol})$ in ethanol $(30 \mathrm{~mL})$ was refluxed for $3 \mathrm{~h}$. The precipitate that formed while hot was filtered off and recrystallized from dioxane as pale yellow needles. This product was assigned as 9-ethoxycarbonyl-8methyl-10-styrylpyrido[3',2':4,5]|thieno[2,3-e]pyrrolo[1,2-a]pyrazine-4(5H)-one (15); yield: $0.20 \mathrm{~g}$ (24\%); mp 264-265 ${ }^{\circ} \mathrm{C}$. IR (cm $\left.{ }^{-1}\right): 3243(\mathrm{NH}), 2999,2974\left(\mathrm{C}-\mathrm{H}\right.$, aliphatic), 1719(C=O, ester), 1644 (C=O, pyrazineone). ${ }^{1} \mathrm{H}$ NMR (DMSO- $\left.d_{6}\right): \delta$ 6.20-7.00 (m, $11 \mathrm{H}: \mathrm{CH}=\mathrm{CH}$, pyrrole-H, Ar-H's), 4.48-4.52 (q, 2H, OCH $), 2.71\left(\mathrm{~s}, 3 \mathrm{H}, \mathrm{CH}_{3}\right)$, 1.15-1.18 (t, 3H, CH $\mathrm{CH}_{3}$ of ester). Anal. Calcd. for $\mathrm{C}_{24} \mathrm{H}_{19} \mathrm{~N}_{3} \mathrm{O}_{3} \mathrm{~S}$ (429.11): C, 67.12; H, 4.46; N, 9.78\%. Found: C, 67.39; $\mathrm{H}, 4.48 ; \mathrm{N}, 9.91 \%$.

The filtrate of the above crude product was allowed to cool whereby a yellowish white crystals precipitated. They were filtered off and recrystallized from ethanol. This compound was identified as ethyl 5-ethoxycarbonyl-6-methyl-3-(1H-pyrrol-1-yl)-4-styrylthieno[2,3-b]pyridine-2-carbamate (14); yield: $0.34 \mathrm{~g}(36 \%) . \mathrm{mp}$ $183-184{ }^{\circ} \mathrm{C} . \mathrm{IR}\left(\mathrm{cm}^{-1}\right)$ : $3411(\mathrm{NH}), 3025$ (CH, aromatic), 2976, 2930 (CH, aliphatic), 1728 (C=O, ester), 1669 (C=0, carbamate). Anal. Calcd. for $\mathrm{C}_{26} \mathrm{H}_{25} \mathrm{~N}_{3} \mathrm{O}_{4} \mathrm{~S}$ (475.16): C, 65.67; H, 5.30; N, 8.84\%. Found: C, 65.60; H, 5.38; N, 8.71\%.

\section{References}

1. Henry, G. D. Tetrahedron 2004, 60, 6043.

https://doi.org/10.1016/i.tet.2004.04.043

2. Altaf, A. A.; Shahzad, A.; Gul, Z.; Rasool, N.; Badshah, A.; Khan, E.; Lal, B. J. Drug Design Med. Chem. 2015, $1,1$.

https://doi.org/10.1155/2015/913435

3. Bakhite, E.A. Phosphorus Sulfur Silicon Relat. Elem. 2003, 178, 929. https://doi.org/10.1080/10426500307855

4. Litvinov, V. P.; Dotsenko, V. V.; Krivokolysko, S. G. Russ. Chem. Bull. Intern. Ed. 2005, 54, 864. https://doi.org/10.1007/s11172-005-0333-1

5. Schnute, M. E.; Anderson, D. J.; Brideau, R. J.; Ciske, F. L.; Collier, S. A.; Cudahy, M. M.; Eggen, M.; Genin, M. J.; Hopkins, T. A.; Judge, T. M.; Kim, E. J.; Knechtel, M. L.; Nair, S. K.; Nieman, J. A.; Oien, N. L.; Scott, A.; Tanis, S. P.; Vaillancount, V. A.; Wathen, M. W.; Wieber, J. L.; Bioorg. Med. Chem. Lett. 2007, 17, 3349. https://doi.org/10.1016/j.bmcl.2007.03.102 
6. Attaby, F. A.; Elghandour, A. H. H.; Ali, M. A.; Ibrahem, Y. M. Phosphorus Sulfur Silicon Relat. Elem. 2007, $182,695$.

https://doi.org/10.1080/10426500600887313

7. Bahekar, R. H.; Jain, M. R.; Jadav, P. A.; Prajapati, V. M.; Patel, D. N.; Gupta, A. A.; Sharma, A.; Tom, R.; Bandyopadhya, D.; Modi, H.; Patel, P. R. Bioorg. Med. Chem. 2007, 15, 6782.

8. Abdel-Rahman, A. E.; Bakhite, E. A.; Al-Taifi, E. A. Pharmazie 2003, 58, 372. https://doi.org/10.3184/030823503103174956

9. Hussin, A.M.; Abu-Shanab, F. A.; Ishak, E. A. Phosphorus Sulfur Silicon Relat. Elem. 2000, 159, 55. https://doi.org/10.1080/10426500008043650

10. Sable, P.; Ganguly, S.; Indian J. Heterocycl. Chem. 2014, 23, 245.

11. Mohi El-Deen, E. M.; Abd El-Meguid, E. A.; Hasabelnaby, S.; Karam, E. A.; Nossier, E. S. Molecules 2019, 24, 3650. https://doi.org/10.3390/molecules24203650

12. Sohda, T.; Makino, H.; Baba, A. PCT Int. Appl. WO, 2006, 96, 14, 319.

13. Madhusudana, K.; Shireesha, B.; Naidu, V. G. M.; Ramakrishna, S.; Narsaiah, B.; Rao, A. R.; Diwan, P. V. Eur. J. Pharmacol. 2012, 678, 48.

https://doi.org/10.1016/j.ejphar.2011.12.019

14. Hayakawa, I.; Shioya, R.; Agatsuma, T.; Furukawa, H.; Sugano, Y. Bioorg. Med. Chem. Lett. 2004, $14,3411$. https://doi.org/10.1016/j.bmcl.2004.04.079

15. Kadah, M. S. Int. J. Med. Sci. 2016, 3, 5.

16. Zeng, X. X.; Zheng, R.-L.; Zhou, T.; He, H.-Y.; Liu, J.-Y.; Zheng, Y.; Tong, A.-P.; Xiang, M.-L.; Song, X.-R.; Yang, S.-Y.; Yu, L.-T.; Wei, Y.-Q.; Zhao, Y.-L.; Yang, L. Bioorg. Med. Chem. Lett. 2010, 20, 6282. https://doi.org/10.1016/j.bmcl.2010.08.088

17. Bernardino, A. M. R.; Pinheiro, L. C.; Rodrigues, C. R.; Loureiro, N. L.; Castro, H. C.; Lanfredi-Rangel, A.; Sabtini-Lopes, J.; Borges, J. C.; Carvalho, J. M.; Romeiro, G. A.; Ferreira, F. V.; Fruguphetti, I. C. P. P.; VannierSantos, M. A. Bioorg. Med. Chem. 2006, 14, 5765.

18. Krauze, A.; Germame, S.; Eberlins, O.; Sturms, I.; Klusa, V.; Duburs, G. Eur. J. Med. Chem. 1999, $34,301$.

19. Joshi, K. C.; Chand, P. J. Heterocycl. Chem. 1980, 17, 1783.

20. Furuya, S.; Takeru, N.; Matsumoto, H. Jpn Tokai Tokyyo Koho JP 09169 766, 1997; C. A. 1997, 127,176416

21. Furuya, S.; Choho, N.; Suzuki, N.; Imada, T. PCT Int. Appl. WO 00000 493, 2000; C. A. 2000, $132,64179$.

22. Bridson, P. K.; Davis, R. A.; Renner, L. S. J. Heterocycl. Chem.1985, 22, 753. https://doi.org/10.1002/jhet.5570220328

23. Saito, T.; Yasushi, M.; Sakoshita, M.; Toyda, K.; Shibazalti, T. Eur. Pat. Appl., 1993, 535, 548; C. A. 1993 , 119, 117112.

24. Gad-Elkareem, M. A. M.; Abdel-Fattah, A. M.; Elneairy, M. A. A. Phosphorus Sulfur Silicon Relat. Elem. 2006, 181, 891.

25. Dave, C. G.; Shah, P. R.; Shah, A. B.; Dave, K. C.; Patel, V. J. J. Indian Chem. Soc. 1989, 66, 48.

26. Al-Waleedy, S. A. H.; Bakhite, E. A.; Abbady, M. S.; Abdu-Allah, H. H. M. J. Heterocycl. Chem. 2020, 57, 2379.

27. Mague, J. T.; Mohamed, S. K.; Akkurt, M.; Bakhite, E. A.; Albayati, M. R. IUCr Data 2016 1, x160270. https://doi.org/10.1107/S2414314616008646 